\title{
Hospital and non-hospital costs for fall-related injury in community-dwelling older people
}

\author{
Anne C. Tiedemann ${ }^{\mathrm{A}, \mathrm{B}}$, Susan M. Murray, \\ Bridget Munro $^{\mathrm{A}}$ and Stephen R. Lord ${ }^{\mathrm{A}}$
}

APrince of Wales Medical Research Institute, University of New South Wales

${ }^{\mathrm{B} C o r r e s p o n d i n g ~ a u t h o r . E m a i l: ~ a . t i e d e m a n n @ p o w m r i . e d u . a u ~}$

\begin{abstract}
Objectives: This study determined the cost of fall-related health care in a cohort of community-dwelling people. Methods: 578 people aged 75 years and over were monitored for falls and related health-care costs for one year. Results: The mean cost per injurious fall was $\$ 1600$ (year 2000 dollars). Hospital costs accounted for $67 \%$ of the total cost, even though only $4 \%$ of injurious falls required hospital admission. The cost of non-hospital health care was also substantial. Conclusion: This study highlights the economic burden of falls and the importance of injury prevention strategies.
\end{abstract}

Falls in older people are common, costly and potentially debilitating. Falls are the leading cause of injury-related hospitalisation and death in people aged 65 years and older, and often result in high economic and social costs. ${ }^{1,2}$ Depending on the population under study, between 22 and $60 \%$ of fallers suffer injuries from their falls. ${ }^{3,4}$ Major injuries including soft tissue damage, head trauma, dislocations and fractures occur in 5 to $15 \%$ of all falls in any given year. Furthermore, it has been estimated that $20 \%$ of older people who experience a fall sustain injuries that require medical attention. ${ }^{5,6}$

Studies undertaken in Sweden, the United States and the United Kingdom have drawn attention to the significant direct health-care costs required for the treatment of fallrelated injury. ${ }^{7-12}$ Two recent studies have used aggregated data to examine the current and projected costs of fall injuries in Australia. ${ }^{13,14}$ One study examined all injury categories and found that fall injuries were the most costly of any injury mechanism. ${ }^{13}$ The second study, which was undertaken on behalf of the Australian government, found that ageing of the Australian population in the next 50 years will have a significant impact on the health system due to the increased number of older people suffering fall-related injuries. ${ }^{14}$ The study concluded that prevention strategies will need to deliver a reduction in falls incidence of approximately $66 \%$ in order to maintain cost parity with current health system costs. A third Australian study estimated fallrelated costs in more detail by assessing costs for 79 older people admitted to acute hospital care due to a fall and then discharged to the community. ${ }^{15}$ Community and informal care costs were derived from daily diaries completed by participants in the three-month period following hospital discharge and the results showed that hospital costs accounted for most of the post-fall care costs, but that community and personal costs were also substantial. A larger, subsequent study by the same authors found that more than half of the total fall-related health-care cost was attributed to hospital inpatient care when costs were examined for people attending emergency departments (EDs) in Western Australia over a one-year period. ${ }^{16}$ A limitation of these study designs, however, is that they only included subjects who had attended a hospital ED following a fall-related injury, thereby limiting the focus to the small percentage of falls that result in serious injury.

The current study builds upon the previous populationaggregated cost studies by broadening the scope of costs included in the analysis to include non-hospital health care in addition to costs associated with hospital admission for all injurious falls, regardless of severity. We examined the cost and cost components of all individual injurious falls that occurred in a large sample of community-dwelling people aged 75 years and over, over a one-year period. For each fall we ascertained costs related to hospitalisation, ambulance, ED presentation, non-hospital medical and allied health care, pharmaceutical and diagnostic investigation and other out-of-pocket expenses to provide accurate costs of individual falls and the costs of the various health-care components required for their treatment.

\section{Methods \\ Participants}

The study population comprised community members aged 75 years and over who were randomly drawn from a membership database of a private health insurance 
company and invited to take part in a randomised controlled falls-prevention trial, conducted in northern Sydney between 1999 and 2002. ${ }^{17}$ Exclusion criteria included minimal English, blindness, Parkinson's disease or a Short Portable Mental Status Questionnaire (SPMSQ) score $<7$. People were also excluded from further participation in the study if, after initial assessment, they were found to have a low falls risk (as measured by the Physiological Profile Assessment), since the aim of the trial was to test an intervention for high-risk fallers. ${ }^{18}$ Six hundred and twenty people were enrolled in the study; however, during the study year, 42 people were lost to follow-up due to ill-health, death, leaving the study area or withdrawal of consent. Thus, 578 participants aged between 75 and 98 years (mean $=80.2$; SD = 4.4) were included for cost-of-falls analysis. Informed consent was obtained from all participants prior to participation and approval was given by the Human Studies Ethics Committee at the University of New South Wales (approval number CEPIHS 98048).

\section{Falls definitions and data collection}

Falls experienced by all participants for a period of 12 months were measured prospectively, using monthly fall calendars. Details of each fall, including the injuries sustained and consequent medical treatment, were collected via telephone interview. Injuries were classified using the International Classification of Diseases, Ninth Revision, Clinical Modification (ICD-9-CM). The participants returned the calendars to the research team at the end of each month and participants who did not return calendars were contacted via telephone.

\section{Health-care use and cost data}

Participants who experienced a fall that resulted in healthcare utilisation were contacted via telephone on a weekly basis to collect information relating to ongoing medical, health-care and associated costs. This follow-up continued until the fall-related treatment had ceased (range 1-20 weeks). Information was collected on all aspects of the participants' medical treatment, including hospital costs, outpatient services from medical practitioners and allied health professionals and the cost of pharmaceutical medications and diagnostic investigations. Costs obtained from subjects were total costs and did not take account of Medicare or health fund rebates. The data were collected between the years 1999 and 2002, and the costs calculated using year 2000 fees and charges.

Hospital costs were based on the Diagnosis Related Group (DRG) allocated to each specific fall-related injury, since the authors did not have access to hospital cost information directly from the source. For cases where the length of stay differed from the average length of stay for each DRG, adjustments to the average ward costs were made, i.e. $\operatorname{cost}=\mathrm{DRG}$ figure $\times$ (patient length of stay $/ \mathrm{DRG}$ average length of stay). Estimates from the NSW Health Services Comparison Data were used to calculate missing costs. ${ }^{19}$

Patients who presented at a hospital ED and were discharged without admission were allocated the average cost of a visit to the ED (\$238 for a principal referral hospital and \$271 for a major metropolitan hospital), plus additional costs for individual services such as pathology (\$170 principal referral and \$95 major metropolitan hospital) and imaging (\$174 principal referral and \$123 major metropolitan hospital), where relevant. These cost estimates were obtained from the NSW Health Services Comparison Data. ${ }^{19}$

The cost of ambulance travel in relation to a fall was also included in the analysis and an average fee for service was used (\$143), which was determined through direct contact with the Ambulance Service of NSW.

Costs for services provided by medical practitioners and allied health professionals were obtained from the participants. Where participants were not able to provide specific information, the standard fee-for-service was used. For medical practitioners, standard fees were obtained from the Medicare Benefits Schedule. ${ }^{20}$ For allied health professionals, the following amounts were used (obtained from the relevant representative professional organisations): physiotherapy and occupational therapy, $\$ 50$ initial visit, \$40 subsequent visit; home nurse, $\$ 50$ per hour.

The cost of fall-related pharmaceuticals was ascertained through the Schedule of Pharmaceutical Benefits. ${ }^{21}$ The cost of associated diagnostic investigations such as x-rays was obtained directly from the participant or, when this was not possible, from the Medical Benefits Schedule. ${ }^{20}$

Other out-of-pocket expenses included in the analysis were the repair of broken glasses, purchase of nonprescription analgesics, installation of safety rails in the home and purchase of bandages and other items for wound protection. The amounts for these items were obtained directly from the study participants.

Costs that were beyond the scope of the study and therefore not ascertained included indirect costs such as informal care from friends and family, transport-related costs such as taxi fares to and from medical appointments, and participant opportunity costs such as time away from work.

\section{Statistical analysis}

Descriptive statistics were used to collate the falls data and compare the incidence of falls and the types of injuries suffered between the men and women in the study group. Average costs were calculated for falls that resulted in 
Table 1. The number of falls according to injury status and receipt of medical treatment in study participants between 1999 and 2002 in Sydney, NSW

\begin{tabular}{lccrrrr}
\hline Fall type & \multicolumn{2}{c}{ Men } & \multicolumn{2}{c}{ Women } & \multicolumn{2}{c}{ Total } \\
& $(n=190)$ & $(\%)$ & $(n=348)$ & $(\%)$ & $(\boldsymbol{N}=538)$ & $(\%)$ \\
\hline Without injury & 105 & 55.3 & 92 & 26.4 & 197 & 36.7 \\
No injury but had medical check-up & 2 & 1.0 & 1 & 0.3 & 3 & 0.6 \\
Injury, no medical attention & 56 & 29.5 & 160 & 46 & 216 & 40.1 \\
Injury, medical attention, excluding hospital & 22 & 11.6 & 74 & 21.3 & 96 & 17.8 \\
Injury, medical attention, including hospital & 5 & 2.6 & 21 & 6 & 26 & 4.8 \\
\hline
\end{tabular}

Table 2. The total and adjusted cost of falls injuries that required hospital admission, treatment at an emergency department or other non-hospital services between 1999 and 2002 in Sydney, NSW

\begin{tabular}{|c|c|c|c|c|c|}
\hline \multirow[t]{2}{*}{ Treatment type } & \multicolumn{5}{|c|}{ Cost $(\$)$} \\
\hline & Average & Minimum & Maximum & $\begin{array}{c}\text { Total } \\
\text { (year 2000) }\end{array}$ & $\begin{array}{c}\text { CPI adjusted total* } \\
\text { (year 2006) }\end{array}$ \\
\hline \multicolumn{6}{|l|}{ Hospital admission $(n=13)$} \\
\hline In-hospital services ${ }^{\#}$ & 10003 & 1530 & 26262 & 130041 & 175165 \\
\hline Non-hospital services & 2328 & 11 & 5200 & 30269 & 40772 \\
\hline \multicolumn{6}{|l|}{ Emergency department** $(n=13)$} \\
\hline Emergency department services & 424 & 147 & 1164 & 5512 & 7425 \\
\hline Non-emergency department services & 756 & 101 & 2371 & 9828 & 13238 \\
\hline Other non-hospital services $(n=96)$ & 203 & 10 & 1759 & 19533 & 26311 \\
\hline Total for all services & & & & 195183 & 262912 \\
\hline
\end{tabular}

hospital attendance and those that resulted in medical attention without hospital attendance. The costs obtained were then adjusted to reflect year-2006 costs by multiplying them by the cumulative change in the Consumer Price Index (CPI) between the years 2000 and 2006. ${ }^{22}$ For this calculation, the figure used was the percentage change of the weighted average of eight Australian capital cities for each year for the CPI health group only. The data were analysed using SPSS 11.5 software. $^{23}$

\section{Results}

In the 12 -month study period, $48 \%$ of the study participants fell one or more times. The total number of falls over this period was 538. In the follow-up year, 303 people (52\%) suffered no falls, 145 (25\%) suffered one fall and $130(23 \%)$ suffered two or more falls. The proportion of men and women in each fall category was similar $\left(\chi^{2}=3.39,3\right.$ d.f., $\left.p=0.34\right)$.

Table 1 illustrates the number of injurious falls that occurred during the study period and the subsequent type of medical treatment that was sought. More women than men suffered an injury $\left(\chi^{2}=18.14,1\right.$ d.f., $\left.p<0.001\right)$ and suffered falls that required medical treatment $\left(\chi^{2}=14.29\right.$, 1 d.f., $p<0.001)$.

Of the 538 falls, a total of 458 injuries occurred, including bruising with intact skin surface (39\% of the injuries), sprains and strains of joints and muscles (17\%) and superficial injuries (15\%). Fractures accounted for $5 \%$ of the injuries, including two hip fractures. Women were more likely to suffer fall-related fractures than men, with $83 \%$ of the fractures occurring in women. Men had a higher rate of open wound injuries, accounting for $33 \%$ of all men's injuries compared with $16 \%$ in women.

Table 2 presents the average, minimal, maximal, total and year-2006-adjusted costs for falls injuries that required inpatient hospital, ED and non-hospital services. The overall cost of fall-related health care for the study population in the follow-up period was $\$ 195183$. Sixty-seven percent of this cost was due to services utilised while in hospital, 3\% was due to services utilised while in an ED and the remaining $30 \%$ was due to services utilised from non-hospital sources. 
Table 3. The total and adjusted cost of falls in study participants according to major injury categories between 1999 and 2002 in Sydney, NSW

\begin{tabular}{|c|c|c|c|c|c|}
\hline \multirow[t]{2}{*}{ Injury category } & \multicolumn{5}{|c|}{ Cost (\$) } \\
\hline & Average & Minimum & Maximum & $\begin{array}{c}\text { Total } \\
\text { (year 2000) }\end{array}$ & $\begin{array}{c}\text { CPI adjusted total* } \\
\text { (year 2006) }\end{array}$ \\
\hline Fracture $(n=23)$ & 5719 & 28 & 30867 & 131529 & 177170 \\
\hline Sprain/strain $(n=36)$ & 656 & 11 & 11382 & 23601 & 31791 \\
\hline Other injuries $(n=62)$ & 646 & 10 & 13480 & 40053 & 53951 \\
\hline
\end{tabular}

The cost of falls for the major injury categories: fractures, sprains and strains, and other injuries are summarised in Table 3. Fractures were the injury type that incurred the greatest medical costs, with the highest overall individual cost of $\$ 30867$ being for a hip fracture. Fractures comprised 5\% of all the injuries suffered, yet accounted for $67 \%$ of the total cost of fall-related injury.

\section{Discussion}

In the 12 -month study period, $48 \%$ of the participants fell one or more times, a rate that would be anticipated for a sample aged 75 years and over, which had excluded people with a low risk of falling. ${ }^{2,3}$ The proportion of falls that resulted in injuries (63\%) and injuries requiring medical care $(23 \%)$ are also consistent with previous research assessing similar populations. ${ }^{2}$

The total cost of fall-related treatment was $\$ 195183$, which equates to a total of $\$ 262912$ using year-2006-adjusted figures. This translates to an average cost of $\$ 1600$ per person for the 122 participants who sustained falls requiring medical care in the study period. This figure is lower than the average costs reported in Australian studies by Potter-Forbes and Aisbett (\$5688), Hall and Hendrie (\$4291) and Hendrie et al. (\$4619). ${ }^{13,15,16}$ The lower cost is most likely due to a greater capture of people who had minor injuries that required treatment only from a medical practitioner or allied health worker: $\$ 1600$ is therefore likely to represent a more accurate estimate of all injurious falls suffered by older people in community. When the analyses were restricted to participants attending the ED (and possible subsequent hospital admission), the average cost was $\$ 6756$. This figure is more comparable to the previous studies that used indirect means for estimating nonhospital care or recruited their study population from ED attendees only. $13,15,16$

Only $4 \%$ of the falls that resulted in injuries led to admission to hospital. Despite this, hospital costs accounted for $67 \%$ of the total cost of injury-related health care. This figure is midway between the findings of Hendrie et al. (53\%), Potter-Forbes and Aisbett (55\%) and Hall and Hendrie (80\%)..$^{13,15,16}$ Again, when only those requiring
ED and hospital care were considered, hospital costs accounted for a similar percentage of total costs $(74 \%)$ as reported by Hall and Hendrie. ${ }^{15}$ These findings confirm that a small minority of falls that result in serious injury are responsible for the bulk of health care costs for this injury mechanism.

The major strengths of the current study were that it was comparatively large, population based and used individual rather than aggregated data. Also, associated costs data were collected prospectively with a rigorous protocol of follow-up interviews until health care utilisation had ceased. This approach is optimal for maximising the recording of all falls and for calculating non-hospital costs, as routine data collection is not undertaken. ${ }^{24}$ The findings indicated that non-hospital costs were substantial and accounted for $19 \%$ of the costs of falls that required admission to hospital and $64 \%$ of the costs of falls requiring treatment from an ED. The overall proportion of costs attributed to non-hospital services for all falls was $30 \%$. These findings, therefore, complement the research undertaken using aggregated data by providing accurate estimates of costs of all sources of health care.

The study also has a number of limitations. Participants were recruited from a private health insurance database and only those classified as 'high risk fallers' were included, so the study population may not be representative of the older population as a whole. It is possible that this could skew the degree and modality of treatment sought for falls injuries. Nevertheless, the findings are similar to recent Australian studies that have assessed costs in general community populations. ${ }^{13,15,16} \mathrm{~A}$ further limitation is that indirect costs were not ascertained. Previous research has shown indirect costs to be quite substantial in relation to fall injury, so the figures from this study do not provide the total extent of the economic burden of falls injuries. ${ }^{13}$

In conclusion, this study provides detailed cost estimates of hospital and non-hospital treatment for fall-related injuries suffered by community-dwelling older people who were at an increased risk of falls. The inclusion of 
treatment from non-hospital services provides a broader estimate of the cost of fall-related injury compared with previous Australian studies. This information may assist health-care planners to guide the allocation of funding priorities and provide estimates of falls-injury treatment categories for use in cost-effectiveness studies of falls prevention strategies.

\section{Acknowledgments}

The National Health and Medical Research Council (POPI Partnership in Injury and Project Grants), and the Medical Benefits Fund of Australia supported this study.

\section{References}

1. Baker SP, Harvey AH. Fall injuries in the elderly. Clin Geriatr Med 1985; 1: 501-12.

2. Tinetti ME, Doucette EJ, Claus E, Maratolli R. Risk factors for serious injury during falls by older persons in the community. J Am Geriatr Soc 1995; 43: 1214-21.

3. Speechley M, Tinetti M. Falls and injuries in frail and vigorous community elderly persons. J Am Geriatr Soc 1991; 39: 46-52.

4. Lord SR. Falls in the elderly: admissions, bed use, outcome and projections. Med J Aust 1990; 153: 117-8.

5. Tinetti M, Williams C. Falls, injuries due to falls and the risk of admission to a nursing home. N Engl J Med 1997; 337: 1279-84. doi:10.1056/NEJM199710303371806

6. Reinsch S, MacRea P, Lachenbruch PA, Tobis JS. Attempts to prevent falls and injury: a prospective community study. Gerontologist 1992; 32: 450-6.

7. Sjogren H, Bjornstig U. Unintentional injuries among elderly people: incidence, causes, severity, and costs. Accid Anal Prev 1989; 21: 233-42. doi:10.1016/0001-4575(89)90014-6

8. Alexander BH, Rivara FP, Wolf ME. The cost and frequency of hospitalization for fall-related injuries in older adults. $\mathrm{Am} J$ Public Health 1992; 82: 1020-3.

9. Covington DL, Maxwell JG, Clancy TV. Hospital resources used to treat the injured elderly at North Carolina trauma centers. J Am Geriatr Soc 1993; 41: 847-52.

10. Carroll NV, Slattum P, Cox F. The cost of falls among the community-dwelling elderly. J Manag Care Pharm 2005; 11: 307-16.

11. Findorff M, Wyman J, Nyman J, Croghan C. Measuring the direct healthcare costs of fall injury event. Nurs Res 2007; 56: 283-7. doi:10.1097/01.NNR.0000280613.90694.b2

12. Scuffham $P$, Chaplin S, Legood R. Incidence and costs of unintentional falls in older people in the United Kingdom. J Epidemiol Community Health 2003; 57: 740-4. doi:10.1136/jech.57.9.740
13. Potter-Forbes M, Aisbett C. Injury costs: A valuation of the burden of injury in New South Wales in 1998-1999. Sydney: NSW Injury Risk Management Research Centre, University of New South Wales; 2003.

14. Moller J. Projected costs of fall related injury to older persons due to demographic change in Australia. Canberra: Commonwealth Department of Health and Ageing; 2003.

15. Hall SE, Hendrie DV. A prospective study of the costs of falls in older adults living in the community. Aust $N Z J$ Public Health 2003; 27: 343-51. doi:10.1111/j.1467842X.2003.tb00405.x

16. Hendrie D, Hall SE, Arena G, Legge M. Health system costs of falls of older adults in Western Australia. Aust Health Rev 2004; 28: 363-73.

17. Lord SR, Tiedemann A, Chapman K, Munro B, Murray SM, Sherrington $\mathrm{C}$. The effect of an individualized fall prevention program on fall risk and falls in older people: a randomised, controlled trial. J Am Geriatr Soc 2005; 53: 1296-304. doi:10.1111/j.1532-5415.2005.53425.x

18. Lord SR, Menz HB, Tiedemann A. A physiological profile approach to falls risk assessment and prevention. Phys Ther 2003; 83: 237-52.

19. NSW Department of Health. NSW Health Services Comparison Data 1999-2000. Volume 2 Case-mix Measures. Sydney: NSW Department of Health; 2003. Available from URL: http://www.health.nsw.gov.au/pubs/2003/pdf/ yellowbook_vol2_99-00.pdf (Cited 26 October 2005.)

20. Australian Government Department of Health and Ageing Medicare Benefits Schedule. 1 November 2000. Canberra: Commonwealth Department of Health and Ageing.

21. Schedule of Pharmaceutical Benefits, August 2000. Canberra: Commonwealth Department of Health and Ageing.

22. Australian Bureau of Statistics. Consumer Price Index. Canberra: Australian Bureau of Statistics; 2008. Available from: http://www.abs.gov.au/AUSSTATS/abs@.nsf/ second + level + view? ReadForm \&prodno $=6401.0 \&$ viewtitle $=$ Consumer\%20Price\%20Index,\%20Australia Sep\%202008 Latest $22 / 10 / 2008 \& \&$ tabname $=$ Past $\% 20$ Future $\% 20$ Issues \& prodno $=6401.0 \&$ issue $=$ Sep $\% 202008 \&$ num $=\&$ view $=\&$ (Cited 3 November 2008.)

23. SPSS Inc. SPSS for Windows release 11.5.0. SPSS Inc: Chicago; 2002.

24. Cummings SR, Nevitt MC, Kidd S. Forgetting falls: the limited accuracy of recall of falls in the elderly. $J$ Am Geriatr Soc 1988; 36: 613-6. 